\title{
Identification of a Non-Linear System Using Volterra Series Model with Calculated Kernels by Legendre Orthogonal Function
}

\author{
Vahid Mossadegh, Mahmood Ghanbari \\ Department of Electrical Engineering, Aliabad Katoul Branch, Islamic Azad University, Aliabad Katoul, Iran
}

\begin{abstract}
Article Info
Article history:

Received Jun 5, 2017

Revised Aug 15, 2017

Accepted Aug 22, 2017

Keyword:
Heat exchanger system
Identification of non-linear
system
Legendre orthogonal function
Optimization
Volterra series

\section{Keyword:}

Heat exchange system Optimization

Volterra series

ABSTRACT

Modeling and identification of non-linear systems have gained lots of attentions especially in industrial processes. Most of the actual systems have non-linear behavior and the first and simplest solution in modeling such systems is to linearize them which in most cases the result of linearization is not satisfactory. In this paper, modeling of non-linear systems is investigated using Volterra series model based on Legendre orthogonal function. Expansion of Volterra series kernels by Legendre orthogonal functions causes a reduction in the number of model parameters; hence, complexity of calculations would be decreased. Besides, if the free parameter is selected properly in these orthogonal functions, error is reduced and convergence speed of parameters is increased which leads to an increase in identification accuracy. In this paper, identification of non-linear system is presented with Volterra series expanded by Legendre function and PSO algorithm is used to calculate the optimum free parameters of Legendre function. Finally, in order to validate the efficacy and accuracy, the proposed algorithm is implemented on a non-linear system i.e. heat exchanger with actual data.
\end{abstract}

Copyright @ 2017 Institute of Advanced Engineering and Science. All rights reserved.

\section{Corresponding Author:}

Mahmood Ghanbari,

Department of Electrical Engineering,

Aliabad Katoul Branch, Islamic Azad University,

Aliabad Katoul, Iran.

Email: ghanbari@aliabadiau.ac.ir

\section{INTRODUCTION}

In all branches of science, modeling and identification is used to identify and analyze what is happening in reality. In the last four decades, modeling of non-linear systems is turned into an active research subject because of the necessity of producing accurate models in different sciences [1]. In theory of nonlinear systems, the term "non-linear" is the definition of a class of systems in which linear approximation is not sufficient then more efficient and effective non-linear model is necessary for the system [1]. There are appropriate and efficient methods accessible for modeling and identifying the linear systems, but there are a few methods for identification and modeling of non-linear systems and researches are still under study about these issues [2]. Some of these methods for non-linear systems are Non-linear Least Squares method, Wiener series, Wavelets, Neural networks, Fuzzy Logic, Genetic Algorithm, Kautz method, Non-linear Time Series Analysis method, Multiple Scales method, KBM method, Homotopy Analysis method and Harmonic Balance method. However, these methods cover a limited part of non-linear systems [2-3]. One of the most comprehensive methods in modeling the non-linear systems is the Volterra series. Volterra series is a powerful mathematical analyzing tool in modeling and identification of non-linear systems [4]. Since the modeling by Volterra series does not referred to the hidden state variables of the system, it is able to identify the dynamic behavior of the system without measuring the state variables [5]. This series is the result of the 
works of an Italian mathematician named Vito Volterra in the last 19th century [7, 8]. There are lots of books and articles in literatures dealing with the identification of systems using Volterra series model in which they try to identify a certain system or present a different method to calculate the kernels of this series. Some of the applications of this model is in modeling of respiratory response, renal auto-regulation and neural plasticity [4]. There are literatures in these issues like [9] in which different kinds of Volterra series to identify non-linear system are investigated. In [2], the application of Volterra series in identification and modeling the synchronous generator is presented. The identification accuracy of the non-linear system based on Volterra series in frequency domain is investigated in [10]. A non-linear analytic and numerical study of integral-differential equation for Volterra series is discussed in [11] while [12] presents the selection of general orthogonal bases for second-order Volterra filter.

In this paper, section 2 presents Volterra series and in section 3, the ways of calculating the kernels or factors of Volterra series are illustrated. Section 3-1 discusses the orthogonal function and section 3-2 expresses the expansion of kernels with orthogonal functions. Heat exchanger system is described in section 4 and the results of the simulation are provided in section 5.

\section{VOLTERRA SERIES}

Volterra series is one of the methods for identification of non-linear systems as a black-box. The main advantage of this series is its generality which too many numbers of non-linear systems can be modeled by that [13]. Volterra series provides a model for behavior of a non-linear system like Taylor series, but there is a difference between these two series. The resulted model by Taylor series is extremely depended on the input in a certain time while in the Volterra series the output of non-linear system is depended on the input in all instants of time [6]. Convolution integral is used for modeling a linear system between input and output, while Volterra series is used to identify and model the non-linear system which is a generalization to the convolution integral [2].

Model response is equal to [14]:

$$
y=y_{0}+y_{1}+y_{2}+y_{3}+\ldots
$$

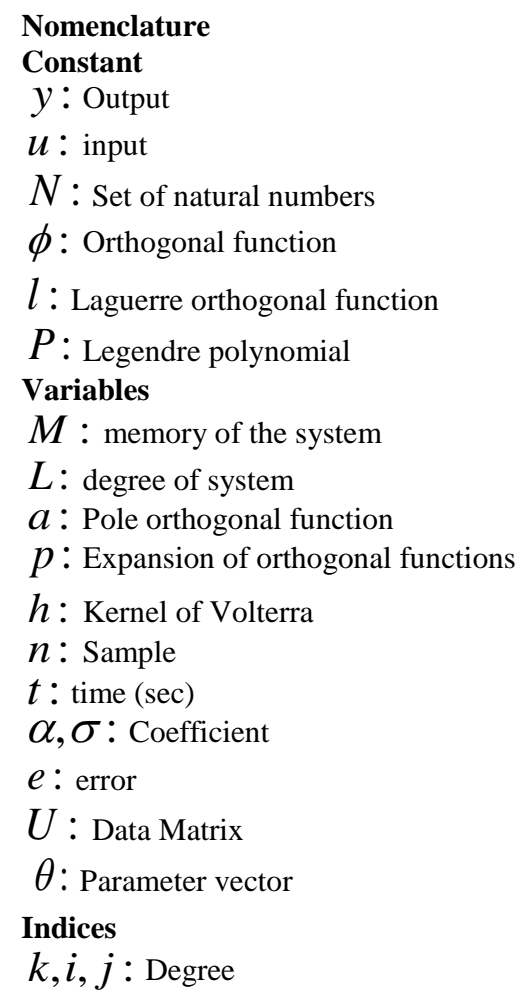
calculated by:

where $\mathrm{y}_{0}$ is a constant value (for example the average of the signal) and the first term is 


$$
y_{1}(n)=\sum_{m_{1}=0}^{\infty} h_{1}\left(m_{1}\right) u\left(n-m_{1}\right)
$$

And high order terms are calculated by the following equations:

$$
\begin{aligned}
& y_{2}(n)=\sum_{m_{1}=0}^{\infty} \sum_{m_{2}=0}^{\infty} h_{2}\left(m_{1}, m_{2}\right) u\left(n-m_{1}\right) u\left(n-m_{2}\right) \\
& y_{3}(n)=\sum_{m_{1}=0}^{\infty} \sum_{m_{2}=0}^{\infty} \sum_{m_{3}=0}^{\infty} h_{2}\left(m_{1}, m_{2}, m_{3}\right) u\left(n-m_{1}\right) u\left(n-m_{2}\right) u\left(n-m_{3}\right)
\end{aligned}
$$

Now, the Volterra series equation is changed into equation (5) by substituting $\mathrm{y}_{\mathrm{i}} \mathrm{s}$ in equation (1):

$$
y(n)=y_{0}+\sum_{m_{1}=0}^{\infty} h_{1}\left(m_{1}\right) u\left(n-m_{1}\right)+\sum_{m_{1}=0}^{\infty} \sum_{m_{2}=0}^{\infty} h_{2}\left(m_{1}, m_{2}\right) u\left(n-m_{1}\right) u\left(n-m_{2}\right)+\ldots
$$

$h_{n}$ is rank $n$ kernel of Volterra. In fact, $h_{1}(t)$ shows impulse function of linear system and $h_{1}(t)$ is impulse function of a one-dimensional non-linear system. In practice, some limitations have to be considered such as too many number of unknowns, limited number of input and output data and the fact that regression matrix is not proper. These deficiencies cause the indefinite series of equation (5) to change into a limited series of equation (6) which means limited degree and memory:

$$
y(n)=y_{0}+\sum_{m_{1}=0}^{M-1} h_{1}\left(m_{1}\right) u\left(n-m_{1}\right)+\sum_{m_{1}=0}^{M-1} \sum_{m_{2}=0}^{M-1} h_{2}\left(m_{1}, m_{2}\right) u\left(n-m_{1}\right) u\left(n-m_{2}\right)
$$

$\mathrm{M}$ is memory of the system and $\mathrm{L}$ is degree of system. Proper selection of these parameters is very effective in speed and accuracy of the identification. In equation (6) there is always $0 \leq(M-1)$ and number of parameters is equal to [15]:

$$
\frac{M(M+1)}{2}+M+1
$$

In order to model the system based on Volterra series, kernels have to fulfill the following features [16]:

For a causal model:

$$
\begin{array}{lc}
g_{n}\left(\tau_{1}, \tau_{2}, \ldots, \tau_{n}\right)=0 \stackrel{\text { if }}{\longrightarrow} \tau_{i} \leq 0 & (i=1, \ldots, n) \\
H_{n}\left(k_{1}, k_{2}, \ldots, k_{n}\right)=0 \stackrel{\text { if }}{\longrightarrow} k_{i} \leq 0 & (i=1, \ldots, n)
\end{array}
$$

For a stable model:

$$
\begin{aligned}
& \sum_{n=0}^{\infty} \int_{\tau_{1}=0}^{t} \ldots \int_{\tau_{n}=0}^{t}\left|g_{n}\left(\tau_{1}, \tau_{2}, \ldots, \tau_{n}\right)\right|<\infty \\
& \sum_{n=0}^{\infty} \sum_{k_{1}=0} \ldots \sum_{k_{n}=0}\left|H_{n}\left(k_{1}, k_{2}, \ldots, k_{n}\right)\right|<\infty
\end{aligned}
$$

Where $\mathrm{g}_{\mathrm{n}}$ is the $\mathrm{n}$-dimensional continuous impulse response and $\mathrm{H}_{\mathrm{n}} \mathrm{n}$-dimensional discrete impulse response. Detailed information about Volterra series and its characteristics is given in [14]. 


\section{CALCULATING THE KERNELS OF VOLTERRA SERIES}

One of the problems of system identification with Volterra series is the estimation of kernels by a pair of input and output which have noise. Calculation of Volterra series factors (kernels) is the main issue in solving the equation and identifying the systems using this series. There are many methods for this purpose such as recursive algorithm [14], Gaussian input method [14], Gradient-based search [17], cross-correlation method [18], method of Hilbert space reproducing kernel [19], and etc. In addition, by expansion of Volterra series with some orthogonal functions, it is possible to reduce the number of parameters and better approximation of them. The main importance of such method is that if we rewrite kernels of Volterra series as an expansion of orthogonal function, series is changed into a linear regression equation which has lower number of parameters with respect to the main series [15]. The main reason for using kernel expansion by orthogonal functions in modeling and identification of the system is to provide a simpler model. Combination of data and knowledge about dynamics of the system by identification processes is very important in using a model with orthogonal functions. By doing this, number of parameters that has to be evaluated are reduced and consequently their evaluation error is decreased which then it increases the accuracy of the model. Another advantage of this procedure is that if orthogonal functions are chosen correctly, they can increase the convergence speed in identification related subjects [20]. Number of kernels is considered symmetrically for reducing the number of parameters:

$$
h_{n}^{\text {sym }}\left(k_{1}, k_{2}, \ldots, k_{n}\right)=\frac{1}{n !} \sum h_{n}\left(k_{1}, k_{2}, \ldots, k_{n}\right)
$$

\subsection{Orthogonal Functions}

Set $\left\{\phi_{n}\right\}_{n \in N}$ is orthogonal in range $[\mathrm{a}, \mathrm{b}]$ if:

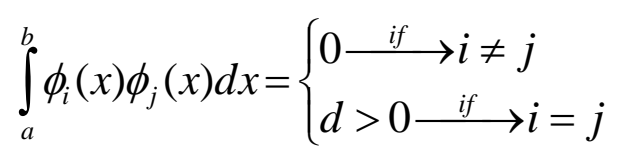

Besides, set $\left\{\Phi_{\mathrm{n}}\right\}_{\mathrm{n}=0}$ is orthogonal to weighing function $\mathrm{w}(\mathrm{x})$ in [a, b] if:

$$
\int_{a}^{b} \phi_{i}(x) \phi_{j}(x) w(x) d x=\left\{\begin{array}{l}
0 \stackrel{i f}{\longrightarrow} i \neq j \\
d>0 \stackrel{\text { if }}{\longrightarrow} i=j
\end{array}\right.
$$

One of the orthogonal function which is widely used in literatures is the Laguerre orthogonal function that is expressed in the Laplace domain [21-24]:

$$
l_{i}(s)=\frac{\sqrt{2 a}}{s+a}\left(\frac{s-a}{s+a}\right)^{i-1}, i \in N
$$

Legendre polynomial is calculated in the continuous time domain with the following equation [21-24]:

$$
P_{i}(t) \cong \sqrt{\sigma(2 i+1)} e^{-.5 \sigma} p_{i}\left(2 e^{-\sigma t}-1\right) \longrightarrow \sigma>0
$$

Legendre polynomial is true in orthogonal condition of equation (12) with respect to weighing function $\mathrm{e}^{-\mathrm{x}}$. Legendre functions are calculated in Laplace domain by considering $a_{i}=\sigma\left(i+\frac{1}{2}\right)$ :

$$
P_{i}(s)=\frac{\sqrt{2 a_{i}}}{s+a_{i}} \prod_{k=0}^{i-1} \frac{s-a_{k}}{s+a_{k}}
$$


Legendre orthogonal functions operate better in approximation of systems with one dominant pole or time constant especially in high frequencies comparing to Laguerre orthogonal functions [22].

Legendre functions have an adjustment parameter named pole that correct and optimal selection of this pole would lead to quicker convergence and increases the accuracy of the system. There are lots of methods for calculating the optimum pole of orthogonal functions such as minimizing the cost function [25], Gram-Schmidt orthogonalization method [26], employing cost function gradient [27]. In this paper, PSO algorithm is used for optimal pole determination by minimizing the criterion function of error squares sum of equation (16).

$$
J=\sum_{n=0}^{N-1}(y(n)-\hat{y}(n))^{2}
$$

where $y(n)$ is the actual output and $\hat{y}(n)$ the estimated output at instant $n$.

\subsection{Expansion of Kernels with Orthogonal Functions}

Now, if the kernels in equation (6) are considered as an expansion of orthogonal functions according to equation (17) and rewrite them, equation (18) is resulted for second-order Volterra [20, 15]:

$$
\begin{aligned}
& h_{1}\left(m_{1}\right)=\sum_{i_{1}=1}^{p} \alpha_{i_{1}} \phi_{i_{1}}\left(m_{1}\right) \\
& h_{2}\left(m_{1}, m_{2}\right)=\sum_{i_{1}=1}^{p} \sum_{i_{2}=1}^{p} \alpha_{i_{1} i_{2}} \phi_{i_{1}}\left(m_{1}\right) \phi_{i_{2}}\left(m_{2}\right) \\
& \ldots \\
& h_{n}\left(m_{1}, \ldots \ldots, m_{n}\right) \sum_{i_{1}=1}^{p} \ldots \sum_{i_{n}=1}^{p} \alpha_{i_{1} \ldots i_{n}} \times \prod_{j=1}^{n} \phi_{i_{j}}\left(m_{j}\right) \\
& \hat{Y}(n)=Y_{0}+\sum_{i_{1}=1}^{p} \alpha_{i_{1}} S_{i_{1}}(n)+\sum_{i_{1}=1}^{p} \sum_{i_{2}=1}^{p} \alpha_{i_{1} i_{2}} S_{i_{1}}(n) S_{i_{2}}(n) \\
& S_{k}(n)=\sum_{m_{1}=0}^{M-1} \phi_{k}\left(m_{1}\right) u\left(n-m_{1}\right)
\end{aligned}
$$

Where the factors are considered symmetrical $\left(\alpha_{i_{1} i_{2}}=\alpha_{i_{2} i_{1}}\right)$, and $\phi_{i}(n)$ is the value of $i_{\text {th }}$ orthogonal functions in point $\mathrm{n}$.

Volterra series is linear toward its factors which this characteristic leads to formulation and calculation of its factors by regression. According to the before-mentioned discussion, equation (18) can be written as a linear regression:

$$
y=U \theta+e
$$

Where $y$ is outputs vector, $\theta$ factors vector, and $e$ noise or error of the model and $U$ known matrix of $\mathrm{S}$ values.

One method to solve the linear regression equations is least square method that on the basis of it, the best parameters which minimize the error are calculated by equation (21) [15]:

$$
\hat{\theta}_{L S}=\left(U^{T} U\right)^{-1} U^{T} y
$$

In some certain conditions, least squares method is not effective to solve the linear regression due to improper condition of data matrix in equation (21). There are some reasons for this:

1. Input is not exciting enough and not able to excite all the modes of the system 
2. The considered rank of model is more than actual rank of system

3. System is identified in closed-loop operation.

For compensating this problem in least square method, inversing $\mathrm{U}^{\mathrm{T}} \mathrm{U}$ is not allowed and alternatively other techniques are employed. In this paper SVD is chosen for this purpose. Besides, equation (22) is a criterion for feasibility assessment of model under the name of residual of normalized output error [28]:

$$
J_{d b}=10 \log \left(\frac{\sum_{n=1}^{M}|\varepsilon(n)|^{2}}{\sum_{n=1}^{M}|y(n)|^{2}}\right)
$$

where $\varepsilon(n)$ is the error between actual and estimated output, $y(n)$ value of actual output at instant $\mathrm{n}$. If identification is performed successfully, this residual has to be contained of noise energy added to the data.

\section{HEAT EXCHANGER SYSTEM}

Case study of this research is a non-linear heat exchanger system. Heat exchangers are used increasingly in most of the industrial processes as an important part of heat and energy regeneration systems such as crude oil preheating, natural gas processing, power plants, refrigeration, exothermic and endothermic reactions and etc. There are different kinds of heat exchangers such as Shell and tube heat exchanger, plate heat exchanger, regenerative heat exchanger and etc. The under study heat exchanger is one of the most applicable heat exchangers which is used in oil refineries and chemical processes [29]. As shown in Figure 1, water is heated by pressurized and saturated steam with a copper tube. In the mentioned system, input variables are the value of fluid flow, temperature of steam and input fluid while output variable is the temperature of output fluid. In order to identify the under study system, input and output data are needed which are measured by a research center in real condition [30]. According to this data, which is composed of 4000 samples of input and output with sampling time of 1, identification is performed. It is clarified that input is the value of fluid flow and output is the temperature of output fluid and in the process of data measurement, temperature of steam and input fluid are considered constant and nominal. Figure 1 shows the heat exchanger of saturated fluid steam.

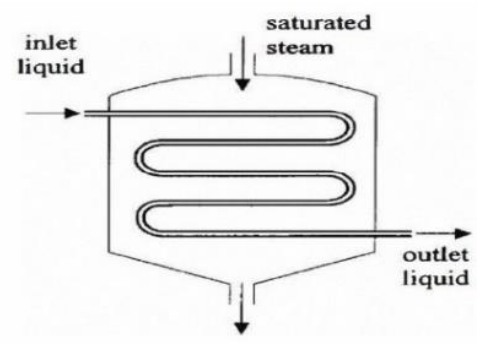

Figure 1. Heat Exchanger of Saturated Fluid Steam

\section{SIMULATION}

As mentioned in section 3-1, at first, values of L (degree of system), p (number of sentence of orthogonal functions) $\mathrm{M}$ (memory of system) and a (pole of orthogonal function) where by them square sum of errors is calculated from equation (16). In this paper, PSO algorithm is used to calculate the free parameters of the system in which the range of free parameters is considered $1 \leq p \leq 14,0<a \leq 5,10 \leq M \leq 400$ for $\mathrm{L}=3$. If the resulted error is not fulfilling, degree of model has to be increased. Input and output data of system are categorized into two groups: one for learning which the model is extracted from it and the second one for experimenting the model. Optimum value of free parameters based on the expansion by orthogonal Laguerre function and orthogonal Legendre function are given in Table 1 for 2500 learning and 500 sampling data. 
Table 1. Optimum Value of Free Parameters Based on the Expansion by Different Laguerre and Legendre Functions for $\mathrm{L}=3$

\begin{tabular}{cccc}
\hline The optimal values with PSO algorithm & Orthogonal Function \\
\hline $\mathrm{M}=200$ & $\mathrm{P}=10$ & $\mathrm{a}=1$ & Laguerre \\
$\mathrm{M}=200$ & $\mathrm{P}=8$ & $\mathrm{a}=0.24$ & Legendre \\
\hline
\end{tabular}

Now, feasibility of the model is tested using the sampling data. Output value of the model and actual output of the system would be like Figure 2 by putting optimum value of Table 1 in Volterra series of equation (18). Figure 3 shows the error of identified model output and actual system.

Table 2 presents numerical value of normalized output error according to equation (22) and sum of error squares according to equation (16) in order to perform a numerical comparison for results of identified heat exchanger system using the expansion by orthogonal Laguerre and Legendre functions.

Table 2. Numerical comparison using the expansion by orthogonal Laguerre and Legendre functions

\begin{tabular}{ccc}
\hline Sum of squared errors & residual of normalized output error $(\mathrm{dB})$ & Orthogonal Function \\
\hline 17.8522 & -124.6064 & Laguerre \\
13.1708 & 127.6456 & Legendre \\
\hline
\end{tabular}

As shown, the proposed method which is the expansion of the Volterra series model by Legendre orthogonal function has lower prediction and sum of squares error comparing to the conventional methods with Laguerre orthogonal function. Besides, as shown in Table 1 for expansion with Legendre orthogonal function, with less number of orthogonal function sentences (that shows the simplicity of the model) better solution is acquired comparing to the Laguerre orthogonal function. This validates the superiority of the proposed method with respect to the conventional method.

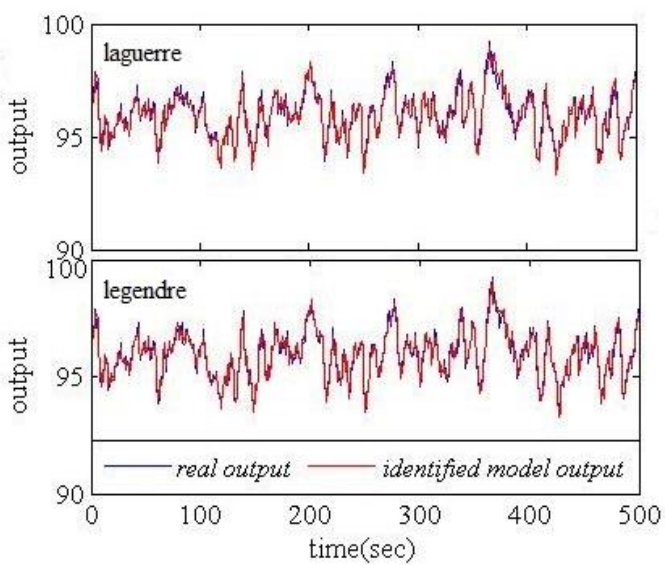

Figure 2. Output of the Identified Model And Actual Output for Optimum Values of Table 1

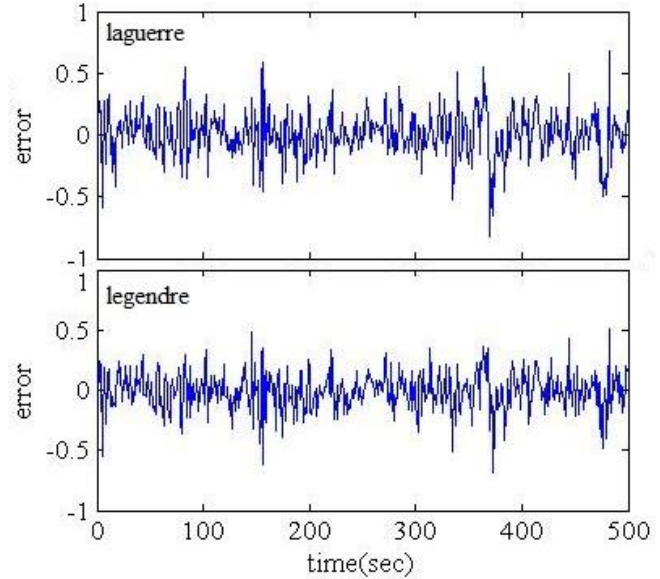

Figure 3. The Error of Identified Model Output and Actual Output

\section{CONCLUSION}

In this paper, it was tried to build a powerful and comprehensive model to identify non-linear systems based on Volterra series and Legendre orthogonal function. Kernels of Volterra series were rewritten as an expansion of orthogonal functions to decrease the complexity and increase the identification accuracy. Optimum value for pole of orthogonal functions, expansion number of orthogonal functions and also the memory of the system were calculated by PSO algorithm. Considering the features of Legendre orthogonal function, identification was performed using this function and the results were compared to the conventional Laguerre function and it was observed that identification with expanded Volterra series by Legendre function has less error and more accuracy with less number of orthogonal function sentences. Besides, in order to improve the result of identification, there are recommendations such as using other orthogonal functions, 
using different optimization methods to better tune the parameter of the orthogonal functions that will be performed in the future researches.

\section{REFERENCES}

[1] Suleiman W, Monin A. New method for identifying finite degree Volterra series. Automatica 2008; 44(2): 488-497.

[2] Fard R D, Karrari M., Malik O P. Synchronous Generator Model Identification for Control Application using Volterra Series. IEEE Trans. Energy Convers 2005; 20 (4):852-858.

[3] Cheng C M, Peng Z K, Zhang WM, Meng G. Wavelet basis expansion-based Volterra kernel function identification through multilevel excitations. Nonlinear Dynamics 2014; 76(2):985-999.

[4] Asyali M H, Juusola M. Use of Meixner functions in estimation of Volterra kernels of nonlinear systems with delay. IEEE Trans. Biomed Eng 2005; 52(2):229-237.

[5] Minu K K, Jessy John C. Volterra Kernel Identification by Wavelet Networks and its Applications to Nonlinear Nonstationary Time Series. Jornal of Information and Data Management 2012; 1(1):4-9.

[6] Parker R S, Heemstra D, Doyle III F J, Pearson R K. The identification of nonlinear models for process control using tailored plant-friendly input sequences. Journal of Process Control 11 2001; 11(2):237-250.

[7] Volterra V. Theory of Functional and of Integral and Integro-differential Equations. Dover, edition 2, New York, 1958.

[8] Volterra V. Sopra le Funzioni che Dipendono de Altre Funzioni. Rend. R.Academia dei 1887;4(3),141-146.

[9] Schmidt C A, Biagiola S I, Cousseau J E, Figueroa J L. Volterra-type models for nonlinear systems identification. Applied Mathematical Modelling, ScienceDirect, 2014; 38(9):2414-2421.

[10] Vitaliy P, Aleksandr F, Sergey P, Yuriy G. Identification Accuracy of Nonlinear System Based on VolterraModel in Frequency Domain. AASRI Conference on Intelligent Systems and Control, ScienceDirect, 2013;4:297-305.

[11] Guebbai H, Aissaoui M Z, Debbar I, Khalla B. Analytical and numerical study for an integro-differential nonlinear Volterra equation. Applied Mathematics and Computation, 2014; 229:367-373.

[12] Kibangou A Y, Favier G, Hassani M M. Selection of generalized orthonormal bases for second-order Volterra filters. Signal Processing, 2005;85(12):2371-2385.

[13] Mahmoodi S, Montazeri A, Poshtan J, Jahed Motlagh M R, Poshtan M. Volterra-Laguerre modeling for NMPC. Signal Processing and Its Applications, ISSPA, 2007:1-4.

[14] Doyle F J, Pearson R K, Ogunnaike B. Identification and Control Using Volterra Models. Springer 2001.

[15] Moodi H. Modeling and Identification of Nonlinear Systems Using Volterra Series and based on Orthogonal Basis Function. M. Sc. Thesis, Sharif University of Technology Electrical Engineering Faculty, Iran, 2008.

[16] da Silva S. Nonlinear Mechanical System Identification Using Discrerte-Time Volterra Models And Kautz Filter. Proceedings of the 9th Brazilian Conference on Dynamics Control and their Applications, 2010:299-305.

[17] Suleiman W, Monin A. New Method for Identifying Finite Degree Volterra. Automatica, 2008;44(2):488-497.

[18] Lee Y W, Schetzen M. Measurement of the Wiener kernels of a non linear system by cross correlation. Int J.Control, 1965;2(3):237-254.

[19] Dodd T J, Harrison R F. A new solution to Volterra series estimation. Proc. IFAC World Congress, 2002.

[20] Rosa A, Campello R J G B, Amarala W C. Choice of free parameters in expansions of discrete-timeVolterra models using Kautz functions. Automatica, 2007;43(6):1084-1091.

[21] Ghanbari M, Haeri M. Parametric identification of fractional-order systems using a fractional Legendre basis. Proc. IMechE, Part I: J. Systems and Control Engineering, 2010:224(I3):261-274.

[22] Ghanbari M. Systems Identification Based on Fractional Orthogonal Functions. Ph.D. thesis, , Islamic Azad University Of Science and Research Of Tehran, Iran, 2009.

[23] Carini A, Cecchi S, Gasparini M, Sicuranza G.L. Introducing Legendre nonlinear filters, in Proc. Of IEEE International Conference on Acoustic, Speech and Signal Processing (ICASSP), Italy, 2014.

[24] Nikolaou M, Hanagandi V. Nonlinearity Quantification and its Application to Nonlinear System Identification, Journal: Chemical Engineering Communications - CHEM ENG COMMUN, vol. 166, no. 1, pp. 1-33, 1998.

[25] Ricardo J G B, Campelloa B,Wagner C, Amaralb D, Favier G. A Note on the Optimal Expansion of Volterra Models Using Laguerre Functions. Automatica, 2006;42(4):689-693.

[26] Kibangoua A Y, Faviera G, Hassanib M M. Selection of Generalized Orthonormal Bases for Second-Order Volterra Filters. Elsevier, Signal Processing, 2005; 85(12):2371-2385.

[27] Hacioglu R, Williamson G A. Reduced Complexity Volterra Models for Nonlinear System Identification. EURASIP Journal on Applied Signal Processing, 2001; 4:257-265.

[28] Ghanbari M,Haeri M. Order and pole locator estimation in fractional ordersystems using Bode diagram. Signal Processing 2011; 91(2):191-202.

[29] Truong N. Nonlinear Identification of a Liquid-saturated Steam Heat Exchangerusing WSDP Models. 11 th International Conference on Control, Automation and Systems in KINTEX, Korea, 2011:61_65.

[30] DAISY, Database for the Identification of Systems, Process Industry Systems, Liquid-saturated steam heat exchanger, available online at ftp://ftp.esat.kuleuven.be/pub/SISTA/data/process_industry/exchanger.dat.gz. 